\title{
ESTIMATION OF 8th, 10th AND 12th ORDER ODF COEFFICIENTS FROM ELASTIC PROPERTIES IN COLD ROLLED STEEL SHEETS BY ADJUSTMENT OF SINGLE CRYSTAL ELASTIC CONSTANTS
}

\author{
KEI SAKATA, DOMINIQUE DANIEL and JOHN J. JONAS \\ Dept. of Metallurgical Engineering, McGill University, 3450 University St., \\ Montreal, Quebec H3A 2A7 Canada
}

(Received June 6, 1989)

\begin{abstract}
In an earlier paper (Sakata et al., 1989), it was shown that the 4th and 6th order ODF coefficients could be successfully derived from Young's modulus measurements using the elastic energy method. However, the values of some of the coefficients fell beyond the expected error ranges. In this study, more appropriate single crystal elastic constants are selected by means of a fitting procedure. Then the ODF coefficients are again estimated in the manner described previously. As a result, the values of the $C_{4}^{11}, C_{6}^{11}, C_{6}^{12}$ and $C_{6}^{14}$ coefficients, which were somewhat inaccurate in the previous calculation, are improved considerably. The volume fractions of the principal preferred orientations are then employed to predict the 8th order coefficients and the fiber components of the $l=10$ and $l=12\left(C_{10}^{11}\right.$, $C_{12}^{11}$ and $\left.C_{12}^{21}\right)$ coefficients. With the aid of the coefficients obtained in this way, both pole and inverse pole figures are drawn, which are in better agreement with those based on X-rays than when only the 4th order coefficients are employed.
\end{abstract}

KEY WORDS ODF coefficients, single crystal elastic constants, elastic energy method, pole figures, cold rolled steel sheet.

\section{INTRODUCTION}

In an earlier paper (Sakata et al., 1989), a method was described for the calculation of the 4th and 6th order ODF coefficients from the elastic properties of cold rolled steel sheets. The elastic energy method (Bunge, 1974) was employed, together with the decomposition of the texture into the principal preferred orientations and their gaussian spreads. The ODF coefficients obtained in this way were often shifted systematically from the $x=y$ lines representing equivalence with those determined from X-ray measurements. Since the uncertainties associated with Young's modulus and X-ray measurements lead primarily to scatter, the systematic shifts in the elastically derived $C_{4}^{11}, C_{6}^{11}, C_{6}^{12}$ and $C_{6}^{14}$ coefficients can be attributed to another source, in this case the assumed values of the single crystal elastic stiffnesses/compliances that play such an important role in elastic calculations regarding polycrystalline materials. According to a recent review (Ledbetter and Reed, 1973), the elastic constants of iron single crystals determined by a number of investigators lie in the following ranges: $c_{11}^{0}=209$ $241, c_{12}^{0}=114-146$ and $c_{44}^{0}=111-119 \mathrm{GPa}$. The selection of inappropriate values 
within these ranges can lead to considerable error in the prediction of the elastic properties of polycrystals, and thus in turn of the ODF coefficients derived from the measurement of these properties.

Another factor which can induce errors in the predicted elastic properties of polycrystals is the assumed grain interaction model. Normally, the Hill (1952) mathematical averaging procedure is employed, which leads to estimates between those of the constant strain (Voigt, 1928) and constant stress (Reuss, 1929) assumptions. However, in heavily cold rolled materials, the $C_{4}^{12}$ coefficients, which are associated with 0 and $180^{\circ}$, i.e. two-fold, anisotropy, calculated with the aid of the Hill assumption are shifted significantly from those obtained from $\mathrm{X}$-rays (Bunge et al., 1970). This has been interpreted as arising from the inappropriateness of employing the Hill assumption for the elongated grain shapes observed along the rolling direction. Furthermore, batch annealed aluminum killed steels often exhibit pancaked grain structures, which may lead to similar inaccuracies. Nevertheless, in our study, no dependence of the $C_{4}^{12}$ coefficients on grain shape was detected.

It was therefore concluded that the adjustment of the single crystal elastic constants within the expected experimental error ranges was permissible so as to match the elastically calculated ODF coefficients with those obtained from $\mathrm{X}$-rays. For this purpose, the sensitivity of the three 4th order ODF coefficients to the values of the single crystal elastic constants was first analyzed. A new set of elastic constants was then selected, and the elastic energy calculation was repeated, leading to better agreement with the X-ray based coefficients than was reported in the previous paper. With the aid of the volume fractions of the main orientations and their gaussian spreads, the $l=8$ and $C_{10}^{11}, C_{12}^{11}$ and $C_{12}^{21}$ coefficients were calculated as well; from these, the so-called "elastic" pole figures could be evaluated more accurately than when solely the 4th order coefficients are employed in the classical manner.

\section{MATERIALS AND EXPERIMENTAL PROCEDURES}

The same 18 commercial cold rolled and annealed steel sheets were employed as in the previous study (Sakata et al., 1989). These consisted of 5 different types of steel: (i) batch annealed aluminum killed drawing quality (AKDQ), (ii) commercial grade rimmed, (iii) high strength low alloy (HSLA) and (iv) and (v) two types of interstitial-free extra low carbon deep drawable grades. The $r$-values for the first type of IF steel (IF1) fell in the range 1.5 to 1.9 (like the AKDQ steels) and the $\Delta r$ 's lay between 0.2 and 0.4 . The second type (IF2) had $r$-values over 2.0 and much smaller $\Delta r$ 's of -0.2 to 0.2 . The latter steels were characterized by a well-developed $\{111\}$ ( $\gamma$-fiber) texture, and by the presence of a significant amount of the $\{554\}\langle 225\rangle$ component (see Figure 9 of the previous paper).

The values of Young's modulus measured by means of Modul-R equipment and the ODF coefficients determined from X-ray data quoted in the earlier paper were employed without modification. Literature data for Young's modulus and the ODF coefficients of an AKDQ steel (Bunge and Roberts, 1969) were also used in the calculations for comparison purposes. 


\section{EFFECT OF THE SINGLE CRYSTAL ELASTIC CONSTANTS ON THE 4th ORDER ODF COEFFICIENTS}

From the preceding 4 th order $\left(C_{4}^{11}, C_{4}^{12}\right.$ and $\left.C_{4}^{13}\right)$ and $C_{6}^{11}$ coefficient results, it was evident that the calculated $C_{4}^{11}$ values were about 0.35 lower on average than those determined from X-rays; a similar tendency was observed for the $C_{6}^{11}$ coefficients. The deviation from the $x=y$ line in each of these cases was beyond the estimated error range associated with the uncertainties in the Young's modulus and X-ray measurements. Furthermore, even the trend lines for the calculated $C_{4}^{12}$ and $C_{4}^{13}$ coefficients were shifted slightly from $x=y$ lines, although these remained within the allowable error ranges. In what follows, it is assumed that the systematic $C_{4}^{11}$ and $C_{6}^{11}$ deviations can be attributed solely to inaccuracies in the values employed for the single crystal elastic constants, and not to the inapplicability of the Hill assumption.

The effect of changes in the single crystal elastic constants on the three 4th order ODF coefficients is demonstrated in Figure 1 for an AKDQ steel. The contour lines of the left hand diagrams display the effect of varying $c_{12}^{0}$ from 131 to $146 \mathrm{GPa}$ in $3 \mathrm{GPa}$ increments while holding $c_{44}^{0}$ constant. On the right hand figures, $c_{44}^{0}$ is increased from 112 to $120 \mathrm{GPa}$ in $2 \mathrm{GPa}$ steps in the same way, while holding $c_{12}^{0}$ fixed. The results are presented in terms of the difference $(\Delta)$ between the newly calculated ODF coefficient and that obtained from the elastic constants used in the previous paper $\left(c_{11}^{0}=230, c_{12}^{0}=136\right.$ and $\left.c_{44}^{0}=116.5 \mathrm{GPa}\right)$. In both cases, the 6th and 8th order coefficients determined from the X-ray measurements were also employed in the elastic energy calculations.

It is evident that the $C_{4}^{11}$ coefficients are most affected by the values of the constants and that the contribution of each single crystal elastic constant is equally significant. The effect on $C_{4}^{12}$ and $C_{4}^{13}$, on the other hand, is less marked. Furthermore, as can be seen from Figure 1, the values of $C_{4}^{12}$ are mainly affected by $c_{11}^{0}$ and $c_{12}^{0}$ and those of $C_{4}^{13}$ by $c_{44}^{0}$.

It was not, of course, possible to select a set of the three variables $\left(c_{11}^{0}, c_{12}^{0}\right.$ and $\left.c_{44}^{0}\right)$ purely on the basis of reducing the error in the single most sensitive ODF coefficient, namely, $C_{4}^{11}$. Nevertheless, the single crystal values could still be determined uniquely by reducing the errors in the $C_{4}^{12}$ and $C_{4}^{13}$ coefficients as well. In this way, the single crystal elastic constants were adjusted for the set of steels so that nearly one-to-one agreement was obtained for the $C_{4}^{11}$ coefficients, while deviations from the $x=y$ lines were minimized for the $C_{4}^{12}$ and $C_{4}^{13}$ coefficients. The single crystal elastic constants established by this means were: $c_{11}^{0}=233$, $c_{12}^{0}=141$ and $c_{44}^{0}=118.5 \mathrm{GPa}$. These values are now available for trial use on further commercial steels without the necessity of carrying out the current fitting procedure.

\section{ESTIMATION OF THE ODF COEFFICIENTS}

\section{a. 4th and 6th Orders}

With the aid of the new values of the elastic constants cited above, the 4th order $\left(C_{4}^{11}, C_{4}^{12}\right.$ and $\left.C_{4}^{13}\right)$ coefficients and three of 6 th order $\left(C_{6}^{11}, C_{6}^{12}\right.$ and $\left.C_{6}^{14}\right)$ were 

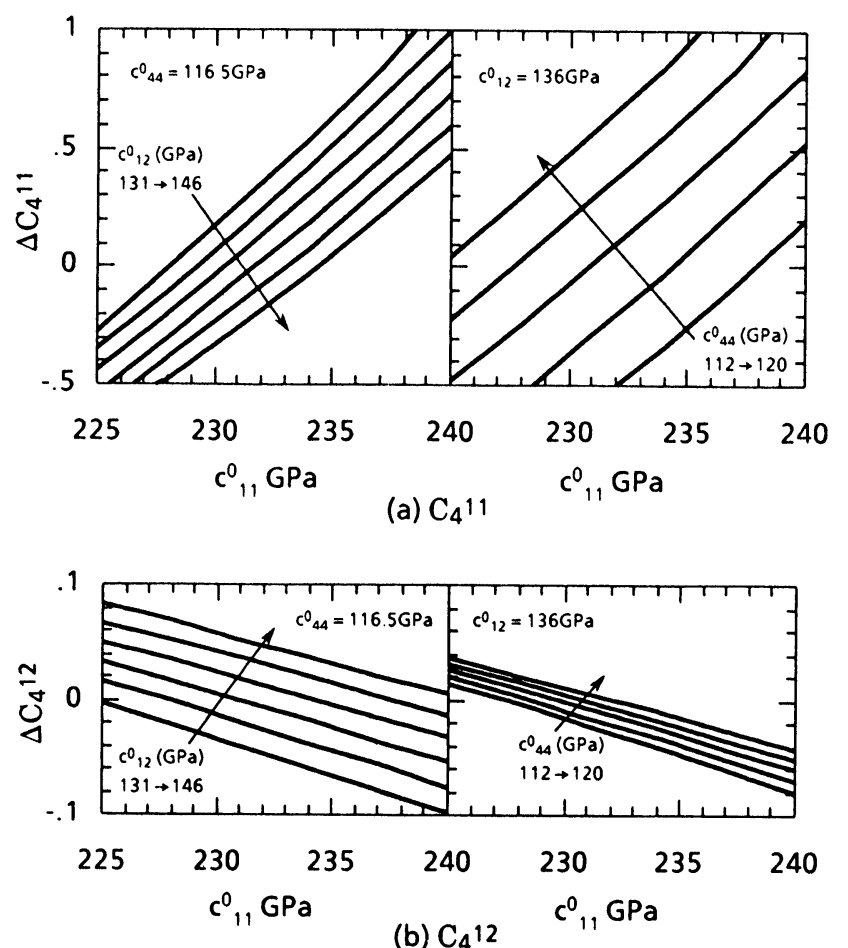

(b) $\mathrm{C}_{4}{ }^{12}$

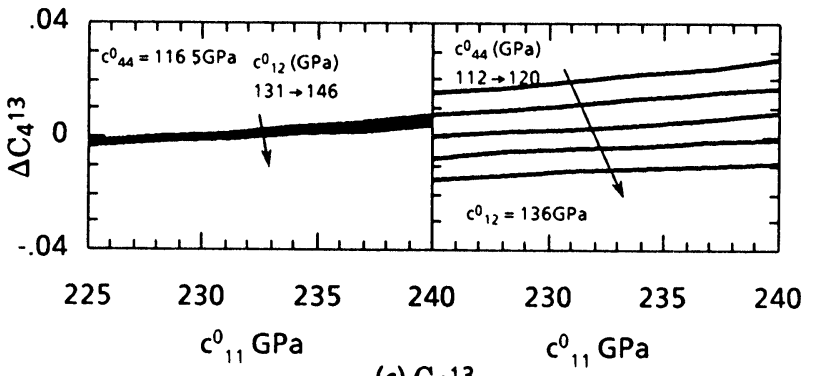

(c) $\mathrm{C}_{4} 13$

Figure 1 Effect of the single crystal elastic constants on the 4th order ODF coefficients in an AKDQ steel: (a) $C_{4}^{11}$, (b) $C_{4}^{12}$ and (c) $C_{4}^{13} . \Delta C_{4}^{11}, \Delta C_{4}^{12}$ and $\Delta C_{4}^{13}$ represent the differences with respect to the values reported in the previous paper. The contour lines in the left hand figures display the effect of increasing $c_{12}^{0}$ in increments of $3 \mathrm{GPa}$ while $c_{44}^{0}$ is held constant. The right hand contour lines concern the analogous effect of increasing $c_{44}^{0}$ in increments of $2 \mathrm{GPa}$ while holding $c_{12}^{0}$ constant.

recalculated by the original method. For the first four coefficients (the three 4th order ones and $\left.C_{6}^{11}\right)$, the volume fractions of $\{111\}\langle u v w\rangle,\{100\}\langle 001\rangle$ or $\langle 011\rangle$ and $\{110\}\langle 001\rangle$ or $\langle 110\rangle$ were taken into account. For the $C_{6}^{12}$ and $C_{6}^{14}$ coefficients, the $\{211\}\langle 011\rangle$ and $\{554\}\langle 225\rangle$ components were also included. In both cases, a gaussian spread of $10^{\circ}$ around these orientations was assumed and, furthermore, a two-to-one volume fraction ratio between the $\{111\}\langle 011\rangle$ and $\{111\}\langle 112\rangle$ components was employed for the $C_{6}^{14}$ calculations, as before.

The 4th order ODF coefficients $C_{4}^{11}, C_{4}^{12}$ and $C_{4}^{13}$, together with $C_{6}^{11}$ obtained in 


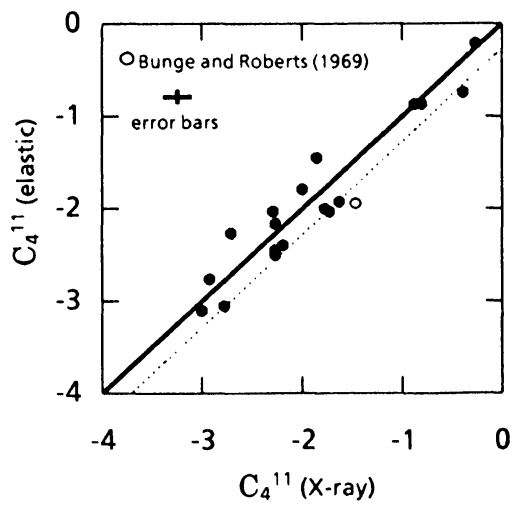

(a)

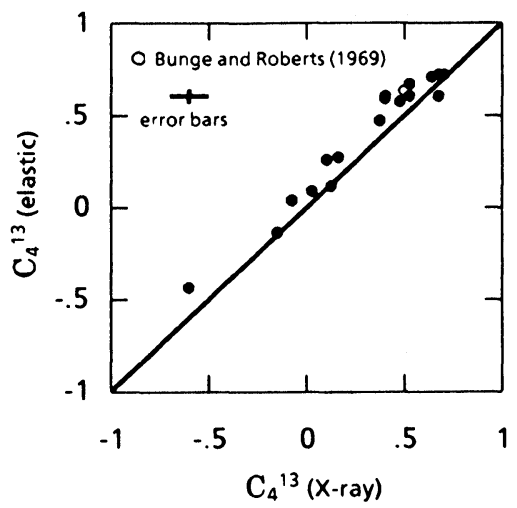

(c)

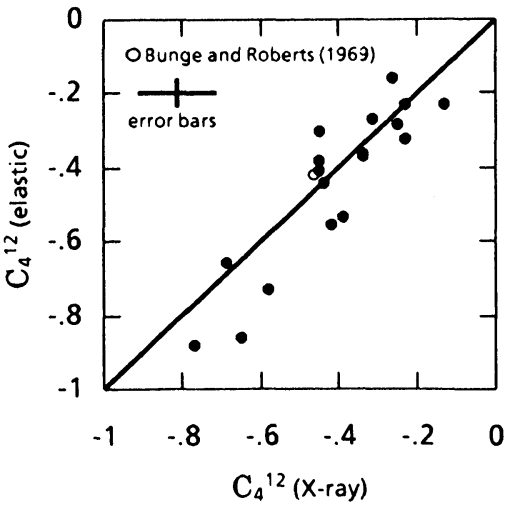

(b)

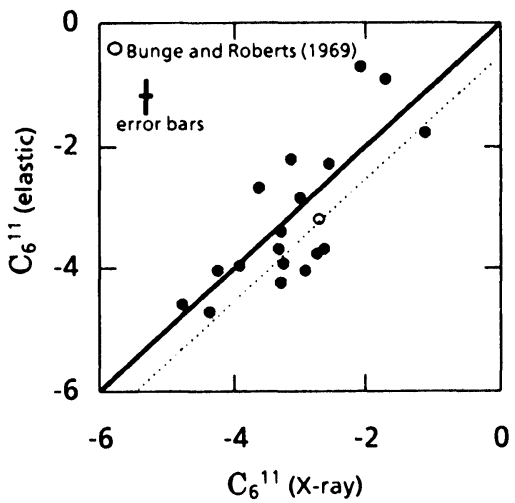

(d)

Figure 2 Correlation between the ODF coefficients estimated in this study and those determined from X-ray measurements: (a) $C_{4}^{11}$, (b) $C_{4}^{12}$, (c) $C_{4}^{13}$ and (d) $C_{6}^{11}$. The dotted lines in (a) and (d) refer to the regressions in the previous report. Here, and in the figures that follow, the symbol (O) refers to values calculated from literature data on an AKDQ steel (Bunge and Roberts, 1969).

this way are compared with those determined from the X-ray measurements in Figure 2. The previous regressions (but not the respective points) are drawn as dotted lines in Figures 2(a) and (d) (and Figure 3, below). It is evident that the newly estimated $C_{4}^{11}$ values coincide very well with those determined by $\mathrm{X}$-ray methods, and the scatter remaining is probably attributable to the experimental errors associated with X-ray measurements and ODF calculations. By contrast, the values of $C_{4}^{12}$ and $C_{4}^{13}$ are less affected by the single crystal elastic constants, and only the former fit is improved slightly. In the case of the $C_{6}^{11}$ calculations, a considerable improvement took place. Since the $C_{6}^{11}$ coefficients are linearly related to the $C_{4}^{11}$ through the ODF coefficients of the $\{111\}$ components (see Figure 3 of the previous paper), this improvement can be linked directly to the improved agreement in the $C_{4}^{11}$ values.

The $C_{6}^{12}$ and $C_{6}^{14}$ coefficients calculated using the elastic energy method are compared with those determined from X-rays in Figure 3. The former (Figure 3a) 


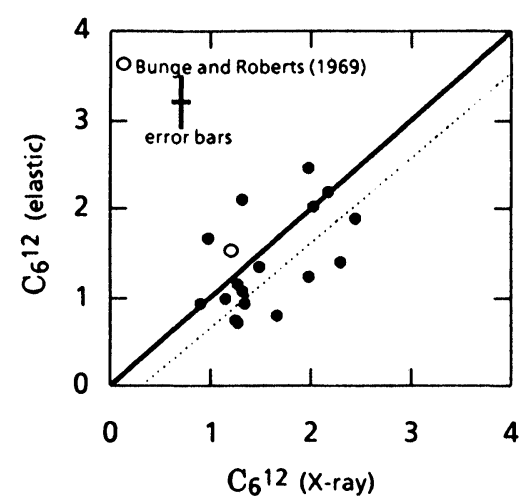

(a)

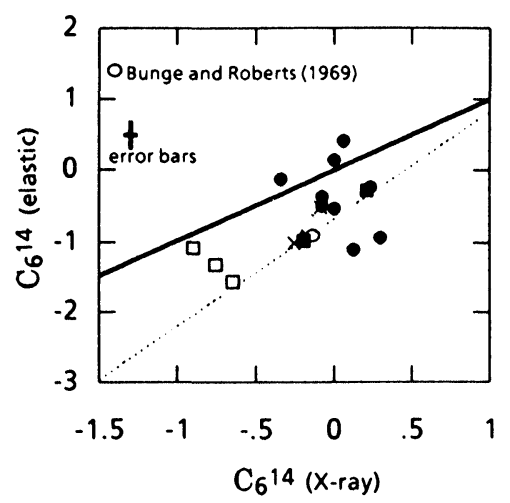

(b)

Figure 3 Comparison of the (a) $C_{6}^{12}$ and (b) $C_{6}^{14}$ coefficients derived in this study and those measured by $\mathrm{X}$-ray methods. The dotted lines are the previous regressions. Legend for Figure 3b: (O)-AKDQ; $(\Delta)$-rimmed; (X)-HSLA; ( $\square)$-IF1; and ( $\square$ )-IF2 steels.

are again closer to the $x=y$ line than in the previous paper, although the amount of scatter is not affected. For the $C_{6}^{14}$ coefficients (Figure $3 \mathrm{~b}$ ), the calculated values fall below the $x=y$ line. Nevertheless, the results for the IF2 steels, which departed considerably from the trend in the last paper, have now improved sufficiently to allow the direct estimation of $C_{6}^{14}$ from elastic measurements.

With respect to $C_{6}^{13}$, as before, the calculated values did not lead to good agreement. This is probably because the $\{100\}$ fiber, which contains both $\{100\}\langle 001\rangle$ and $\{100\}\langle 011\rangle$ components (see Table 2 of the previous paper), was represented by only one of the two components in our calculations. Thus, except for $C_{6}^{13}$, all the 4th and 6th order ODF coefficients were predicted more successfully by the present technique. The introduction of the new values of the single crystal elastic constants can also lead to the more accurate estimation of the volume fractions of the principal preferred orientations, as will be demonstrated below, which may be sufficient for many purposes.

\section{b. Higher Order Coefficients}

As shown in the last paper, the 8th order coefficients determined by X-ray methods are all smaller (in absolute value) than those of the 4th and 6th orders. Thus, they are not particularly useful with respect to improving the accuracy of elastic energy calculations. Nevertheless, they can be employed to increase the precision of texture description in cold rolled steel sheets, for example by leading to more representative pole figures.

The 8th order coefficients calculated by the present method are compared with those obtained from the X-ray measurements in Figure 4. Here, the same volume fractions of the five main components $(\{111\}\langle u v w\rangle,\{100\}\langle 011\rangle$ or $\langle 011\rangle$, $\{110\}\langle 001\rangle$ or $\langle 110\rangle,\{211\}\langle 011\rangle$ and $\{554\}\langle 225\rangle)$ were employed as were used in the $C_{6}^{12}$ and $C_{6}^{14}$ calculations. Furthermore, for the $C_{8}^{14}$ coefficient, which varies along the $\gamma$-fiber with a maximum (7.43) at $\{111\}\langle 112\rangle$ and a minimum $(-7.43)$ at $\{111\}\langle 110\rangle$, the same two-to-one volume fraction ratio between $\{111\}\langle 110\rangle$ 


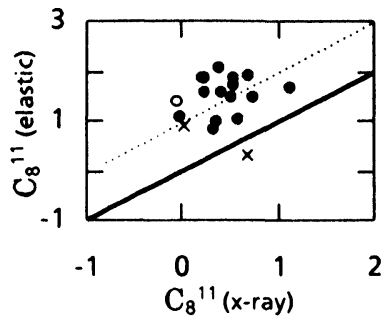

(a)

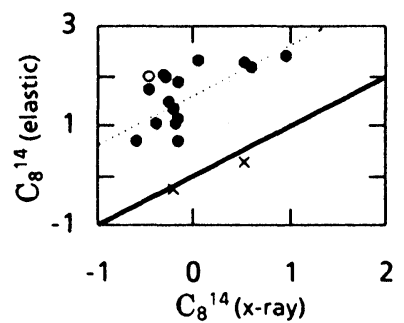

(d)

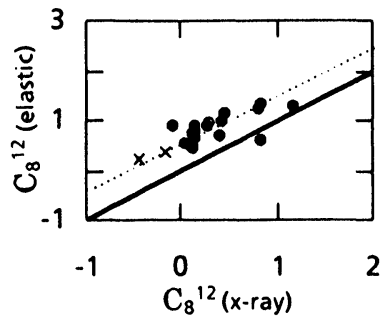

(b)

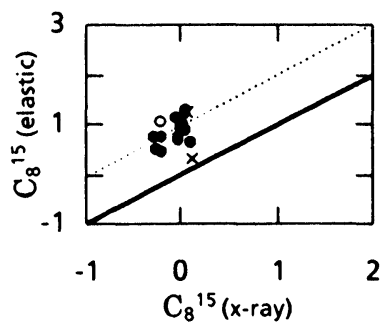

(e)

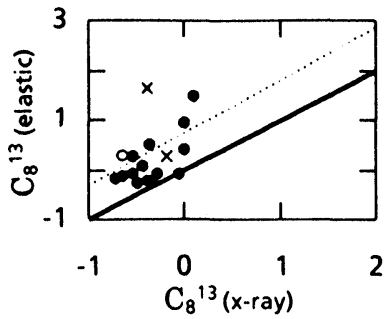

(c)

Figure 4 Comparison of the 8th order ODF coefficients calculated in this study and those derived from X-ray measurements. The dotted lines represent the linear regressions used for the quantitative predictions. The symbols $(X)$ and $(O)$ correspond to the HSLA and other steels, respectively.

and $\{111\}\langle 112\rangle$ was assumed as in the case of the $C_{6}^{14}$ calculation. Except for the $C_{8}^{12}$ coefficients, all the others increase considerably in absolute value. This is probably due to neglect of the minor orientations. Nevertheless, clear trends are evident (except for the HSLA steels), so that the prediction of 8th order coefficients becomes possible using linear regressions.

Finally, some of the 10th and 12th order coefficients, i.e. the $C_{10}^{11}, C_{12}^{11}$ and $C_{12}^{21}$ coefficients, were estimated. These are related to the fiber textures, which are solely functions of the Miller indices $\{h k l\}$ parallel to the rolling plane. Reasonable results, shown in Figure 5, were obtained in all three cases.

From the results described above, it is apparent that the best agreement with the X-ray coefficients is observed when the ODF coefficients display relatively

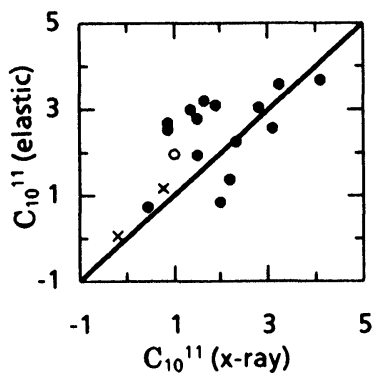

(a)

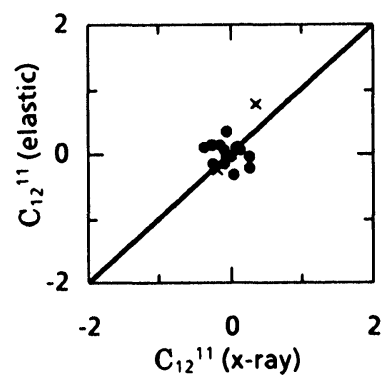

(b)

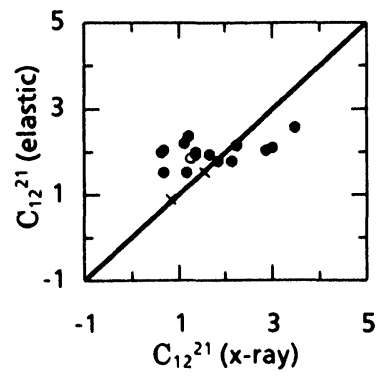

(c)

Figure 5 Comparison of the fiber components of the 10th and 12th order coefficients predicted in this study and those obtained from the X-ray measurements. The symbols $(X)$ and $(O)$ represent the HSLA and other steels, respectively. 
high absolute values. Needless to say, it is the $\{111\}$ orientation that predominates in cold rolled and annealed sheet steels. As a result, those ODF coefficients of the $\{111\}$ orientation which have high absolute values, i.e. $C_{4}^{11}, C_{6}^{11}, C_{10}^{11}$ and $C_{12}^{21}$, are less affected by the uncertainty associated with the neglect of the minor components when the polycrystal coefficients are being estimated.

\section{APPLICATION TO POLE FIGURES DERIVED FROM ELASTIC MEASUREMENTS}

In the previous paper, the 6th order ODF coefficients determined from the elastic measurements were applied to the calculation of the planar distribution of $r$-value. This led to better agreement with the tensile testing results than when solely the 4th order ODF coefficients were employed. In the present study, the inverse pole figures corresponding to the normal (ND, Figure 6) and rolling (RD, Figure 7) directions in an AKDQ steel were calculated for comparison purposes. Here, the 4th and 6th order (except for the $C_{6}^{13}$, see below) and $C_{10}^{11}, C_{12}^{11}$ and $C_{12}^{21}$ ODF coefficients were used without further adjustment, and the 8th order coefficients were estimated using linear regressions. $C_{6}^{13}$ was set to zero instead of using the calculated values.

The results obtained from the coefficients up to $l=12$ (Figures $6 \mathrm{~b}$ and $7 \mathrm{~b}$ ) can be compared with those deduced from just the 4th order coefficients (Figures 6a and $7 \mathrm{a})$ and with those calculated from all the coefficients up to $l=22$ derived from the X-ray measurements (Figures $6 c$ and $7 c$ ). It is evident that the 4th order coefficients alone, which lead to the classical "elastic" pole figures, predict unrealistically low intensities for the (111) poles and even negative values at the (100) position in the ND inverse pole figure. Similarly, nearly isotropic behavior is called for in the RD figure, which is not consistent with the X-ray based results. By constrast, use of the elastically derived ODF coefficients up to $l=12$ leads to must better, and even acceptable, agreement.

The evolution of the inverse pole intensities of the four principal orientations of the ND inverse pole figure is presented in Figure 8 for (a) an AKDQ, (b) a rimmed and (c) an IF2 steel. Here, the $l=22$ results are taken from the $\mathrm{X}$-ray based ODF coefficients. It is apparent that if the ODF coefficients are truncated at an order as low as $l=4$ or 6 , significant errors will appear in the calculated intensities. The employment of the ODF coefficients up to $l=12$, on the other hand, leads to drastic improvements, even though some differences remain with respect to the $\mathrm{X}$-ray based results.

Finally, (200) pole figures are drawn in Figure 9 for one of the AKDQ steels. As for the inverse pole figures presented in Figures 6 and 7, the results derived from the ODF coefficients up to $l=12$ (Figure 9b) can be compared with those obtained solely from the 4th order coefficients (Figure 9a) and with those calculated from the X-ray based coefficients up to $l=22$ (Figure 9c). The 4th order coefficients alone lead to a much weaker $\{111\}$ fiber and to a nearly planar isotropic texture. By contrast, employment of the elastically derived ODF coefficients up to $l=12$ determined in this study leads to considerably closer agreement with the $\mathrm{X}$-ray based results. 


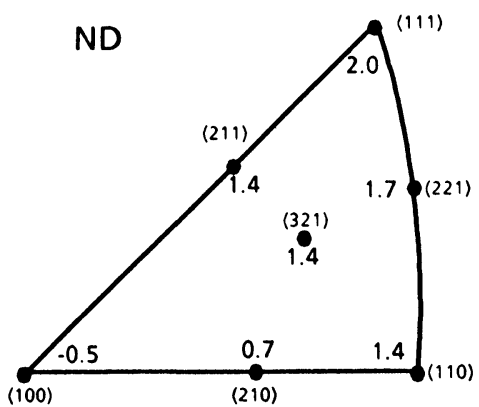

(a) $l=4$

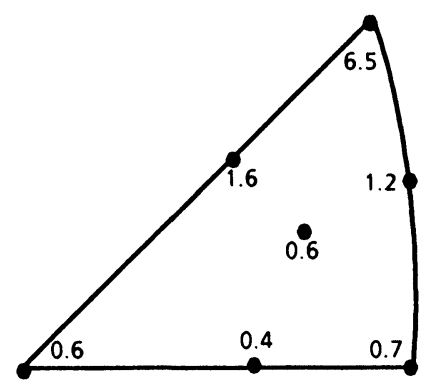

(b) $l=12$

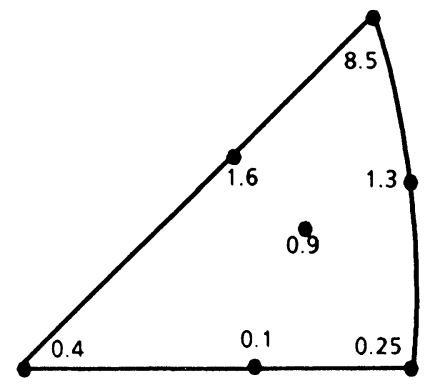

(c) $l=22$

Figure 6 Comparison of inverse pole figures for the normal (ND) direction in an AKDQ steel calculated using (a) only the 4th order coefficients, (b) the coefficients up to $l=12$ derived in this study, and (c) the ODF coefficients up to $l=22$ obtained from X-rays.

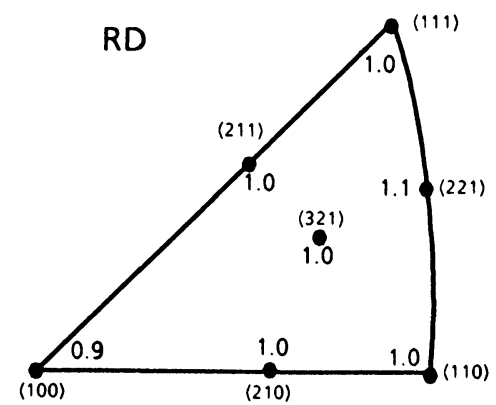

(a) $l=4$

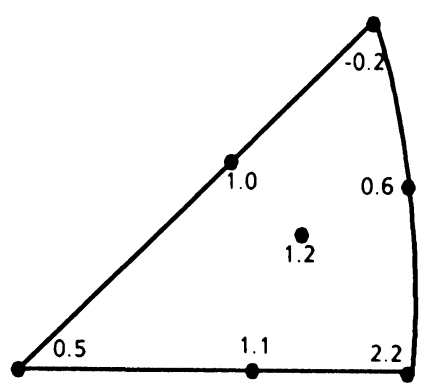

(b) $l=12$

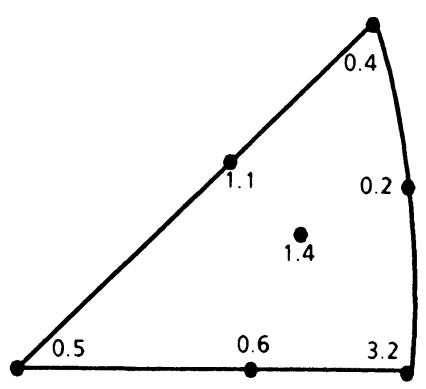

(c) $l=22$

Figure 7 Comparison of inverse pole figures for the rolling (RD) direction in an AKDQ steel calculated using (a) only the 4th order coefficients, (b) the coefficients up to $l=12$ derived in this study, and (c) the ODF coefficients up to $l=22$ obtained from X-rays. 


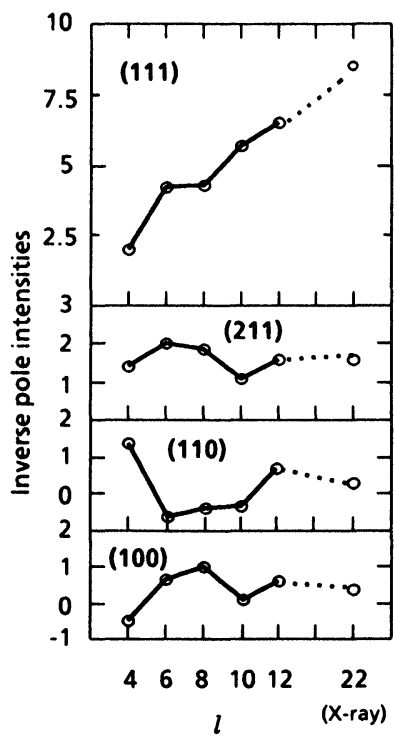

(a) AKDQ

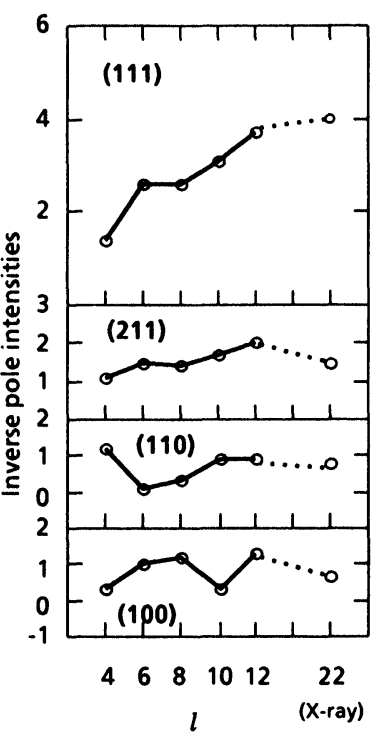

(b) rimmed

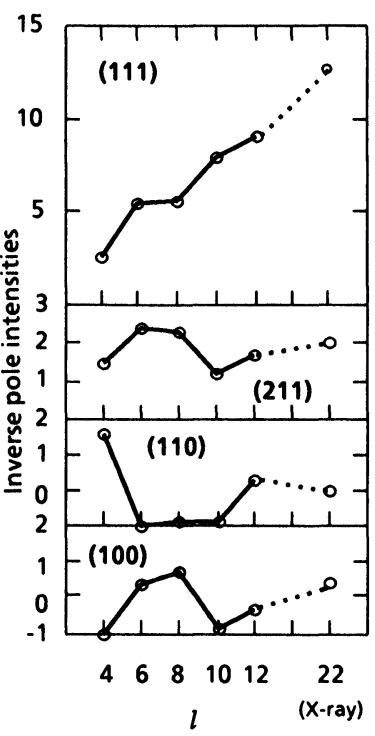

(c) IF2

Figure 8 Evolution of the ND inverse pole intensities for (a) an AKDQ, (b) a rimmed and (c) an IF2 steel as a result of the employment of ODF coefficients of increasing order. The $l=22$ results were calculated from the ODF coefficients determined from X-ray measurements; those for the coefficients up to $l=12$ were derived from the elastic measurements in the present study.

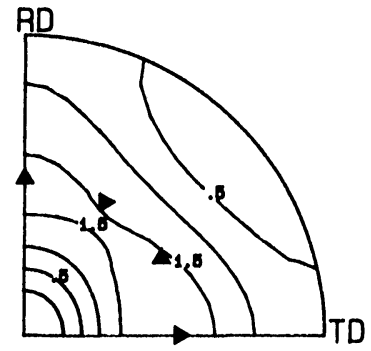

(a) $l=4$

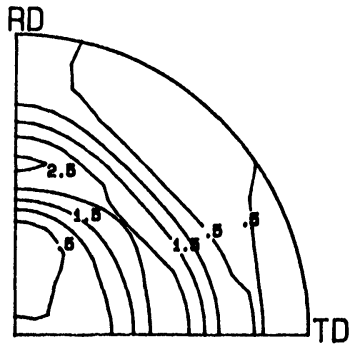

(b) $l=12$

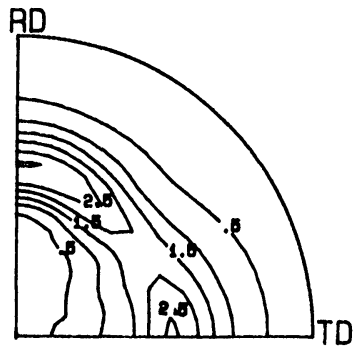

(c) $l=22$

Figure 9 Comparison of (200) pole figures for an AKDQ steel calculated using (a) only the 4th order coefficients, (b) the coefficients up to $l=12$ derived in this study, and (c) the ODF coefficients up to $l=22$ obtained from X-rays. The symbols $(\nabla)$ and $(\Delta)$ represent the locations of the $\{111\}\langle 110\rangle$ and $\{111\}\langle 112\rangle$ components, respectively.

\section{CONCLUSIONS}

In this study, the single crystal elastic constants employed in the elastic energy method were adjusted so that the deviations of the 4th order ODF coefficients from those obtained from X-ray measurements were minimized. The sensitivity of the ODF coefficients to the changes in the single crystal elastic values was first 
examined. It is evident that $C_{4}^{11}$ is affected to a greater degree than $C_{4}^{12}$ or $C_{4}^{13}$. Thus, corrected single crystal values can be obtained by best fitting the $\mathrm{X}$-ray results for the $C_{4}^{11}$ coefficients and then decreasing the deviations corresponding to the $C_{4}^{12}$ and $C_{4}^{13}$ coefficients.

With the aid of the new elastic values, the ODF coefficients of the 4th $\left(C_{4}^{11}, C_{4}^{12}\right.$ and $\left.C_{4}^{13}\right)$ and 6th $\left(C_{6}^{11}, C_{6}^{12}\right.$ and $\left.C_{6}^{14}\right)$ orders were recalculated. Here, the elastic energy method was employed together with the decomposition of the texture into volume fractions of the principal preferred orientations and their gaussian spreads. This procedure leads to considerable improvement in the accuracy of prediction of $C_{4}^{11}$ and of the 6th order coefficients.

The results of the calculations can also be applied to estimating the 8th order coefficients and those of the fiber components for $l=10$ and $12\left(C_{10}^{11}, C_{12}^{11}\right.$ and $\left.C_{12}^{21}\right)$. For the 8th order coefficients, appreciable deviations from the $x=y$ lines are observed. Nevertheless, since the results show clear trends, quantitative estimations can be made using linear regressions. For the $C_{10}^{11}, C_{12}^{11}$ and $C_{12}^{21}$ coefficients, very good agreement is obtained without further adjustment.

Such successful prediction of the high order ODF coefficients up to $l=12$ leads to the derivation of more accurate elastic pole figures than use of solely the 4th order values in the conventional way.

\section{Acknowledgements}

The authors are indebted to Dr. J. F. Bussière of NRCC-IMRI, Boucherville for his encouragement and support, and to M. McLean (Algoma Steel Corp. Ltd.), J. Thomson (Stelco Inc.) and A. Vigeant (Dofasco Inc.) for supplying the steel sheets. They express their thanks to Atomic Energy of Canada Ltd. for the X-ray and neutron diffraction measurements, and to the Canadian Steel Industry Research Association, the Natural Sciences and Engineering Research Council of Canada and the FCAR program of the Ministry of Education of Quebec for financial support. Finally, K. Sakata expresses his gratitude to the Kawasaki Steel Corp. for granting a period of study leave at McGill University as well as for texture measurements and supplying steel sheets.

\section{References}

Bunge, H. J. and Roberts, W. (1969). Orientation distribution, elastic and plastic anisotropy in stabilized steel sheet, J. Appl. Cryst., 2, 116-128.

Bunge, H. J., Öhme, K. and Günther, F. (1970). On the influences of the grain form or the orientation correlation on the Young's modulus of copper sheet, Phys. Stat. Sol. (a), 1, K135-K137.

Bunge, H. J. (1974). The effective elastic constants of textured polycrystals in second order approximation, Kristall Tech., 9, 413-423.

Hill, R. (1952). The elastic behavior of a crystalline aggregate, Proc. Phys. Soc., A65, 349-354.

Ledbetter, H. N. and Reed, P. R. (1973). Elastic properties of metals and alloys, I. iron, nickel and iron-nickel alloys, J. Phys. Chem. Ref. Data, 2, 531-560.

Reuss, A. (1929). Berechnung der fließgrenze von mischkristallen auf grund der plastizitätsbedinung für einkristalle, Z. Angew. Math. Mech., 9, 49-58.

Sakata, K., Daniel, D. and Jonas, J. J. (1989). Estimation of 4th and 6th order ODF coefficients from elastic properties in cold rolled steel sheets, Textures and Microstructures, 11, 41-56.

Voigt, W. (1928). Lehrbuch der Kristallphysik, B. G. Teubner, Leipzig. 